\title{
Characterization of Nanoparticle Films and Structures Using Focused Ion Beam Milling and Transmission Electron Microscopy
}

\author{
C. R. Perrey,* C. B. Carter,* P. G. Kotula,** and J. R. Michael** \\ * Department. of Chemical Engineering and Materials Science, University of Minnesota \\ 421 Washington Avenue S.E., Minneapolis, MN 55455-0132 \\ ** Sandia National Laboratories, Albuquerque, NM 87185-0886
}

In the 1-100 $\mathrm{nm}$ grain-size regime, materials often exhibit dramatically different properties than in bulk materials [1]. These properties have the potential to be used in many new applications and devices. The transmission electron microscope (TEM) can provide the structural and chemical characterization of nanoscale materials at the nanometer scale or better[2]. However, the stringent sample requirements for the TEM can make it difficult or impossible to apply to the investigation of nanoparticle structures and films [3]. The present study investigates the use of the focused ion beam (FIB) tool as an alternative to traditional sample preparation methods for nanoparticle structures.

A "line" of SiC nanoparticles was deposited on a Si wafer. This structure did not adhere strongly to the substrate; the line would flake off if touched lightly with tweezers. Using a FEI Strata DB235 FIB, a TEM sample was prepared using the lift-out (LO) technique $[4,5]$. The samples were then examined in a Tecnai F30 operating at $300 \mathrm{kV}$. The FIB incorporates a scanning electron microscope (SEM) in the FIB chamber, allowing the viewing of the sample during milling. The internal structure of the $\mathrm{SiC}$ line became evident as the milling progressed. Figure 1 shows that the $\mathrm{SiC}$ particles are not uniform in shape and voids are present in the structure, these voids are not caused by mechanical polishing but are intrinsic to the structure of the film A layer of small particles is seen at the line/substrate interface. The LO specimen maintains the structure of the line for TEM examination (Figure 2).

Traditional TEM sample preparation methods do not provide a large electron-transparent area and can induce sub-surface damage, destroying the internal structure of the line. Due to the control of the FIB, the whole LO specimen is available for TEM analysis. Figure 3 is an annular darkfield TEM micrograph, showing no preferential milling at the line/substrate interface. Further TEM investigation yielded important information about numerous structural aspects of the $\mathrm{SiC}$ nanoparticles, such as crystallinity, orientation distributions (Figure 4), defect structures, and sintering behavior. Chemical analyses were performed using energy-filtered imaging (EFI), X-ray energy-dispersive spectrometry (XEDS), and electron energy-loss spectrometry (EELS).

The combination of the FIB and TEM is a powerful tool for the characterization of nanoscale structures. The FIB provides a precise method for preparing specimens thatwould otherwise be challenging to produce, while the incorporation of the SEM allows reattime analysis of the specimen during milling. Subsequent TEM analysis can provide structural and chemical information at the nanometer level or better.

[1] H. Gleiter, Progress in Materials Science 33 (1989) 223.

[2] D. B. Williams and C.B. Carter, Transmission Electron Microscopy, Plenum, NY, 1996.

[3] K. C. Grabar, et al., Analytical Chemistry 69 (1997) 471. 
[4] J. K. Lomness et al., Microscopy and Microanalysis 7 (2001) 418.

[5] J.A. Walraven et al., Proc. of SPIE 4180 (2000) 49.

[6] CRP and CBC were supported by the NSF-NIRT program through grant NSF/DMI-0103169, and acknowledge our colleagues in the University of Minnesota's HPPD program,

particularly W. Mook, A. Gidwani, W. W. Gerberich, J. V. R. Heberlein, S. L. Gershick, and P. H. McMurry for many helpful discussions. JRM and PGK were supported by the United States Dept. of Energy under contract DE-AC04-94AL8500. Sandia is a multiprogram laboratory operated by Sandia Corporation, a Lockheed-Martin company, for the U.S. DOE.

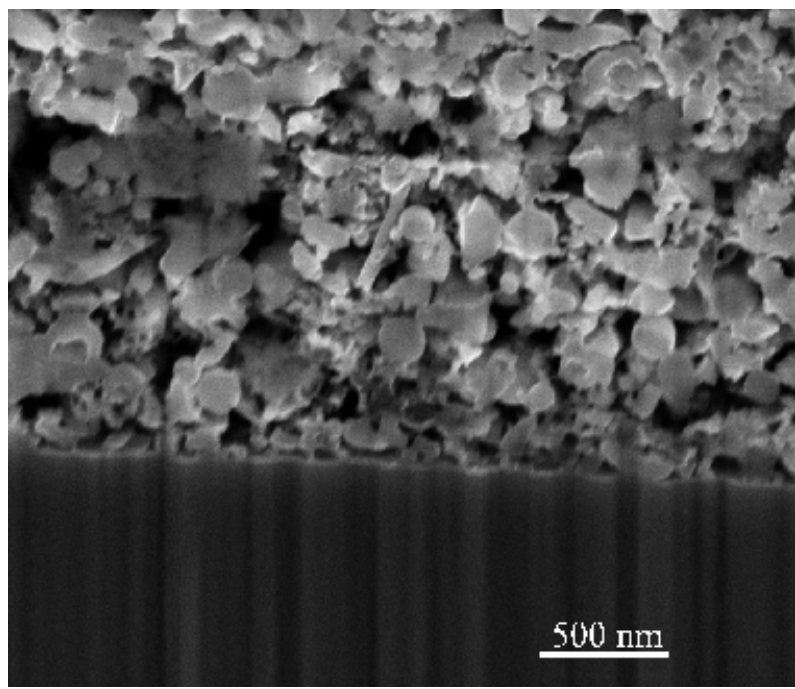

FIG. 1. SEM micrograph showing the varying microstructure of the $\mathrm{SiC}$ nanoparticles and a layer of particles at the line/substrate interface.

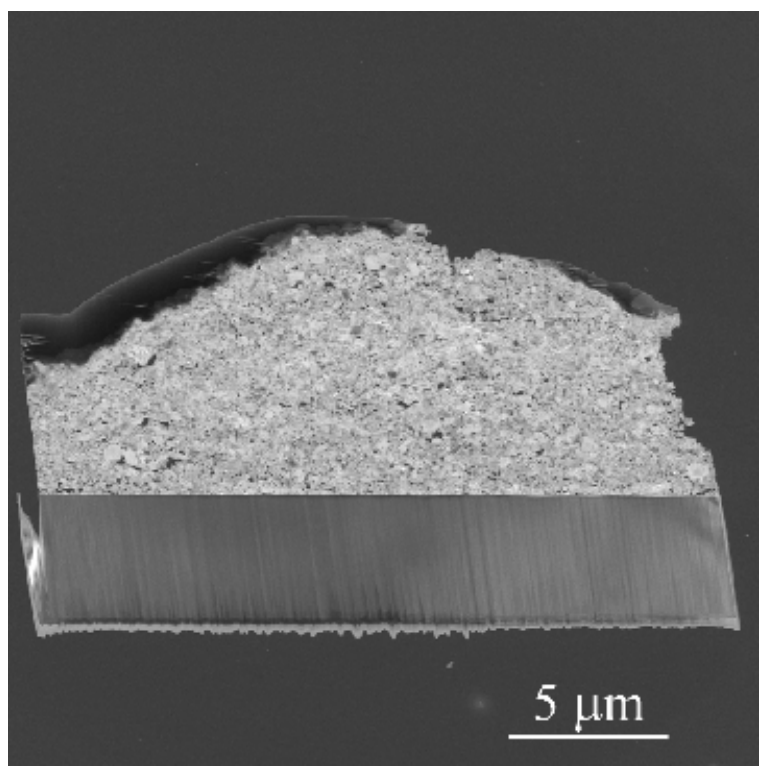

FIG. 3. Annular dark-field TEM micrograph of the LO specimen.

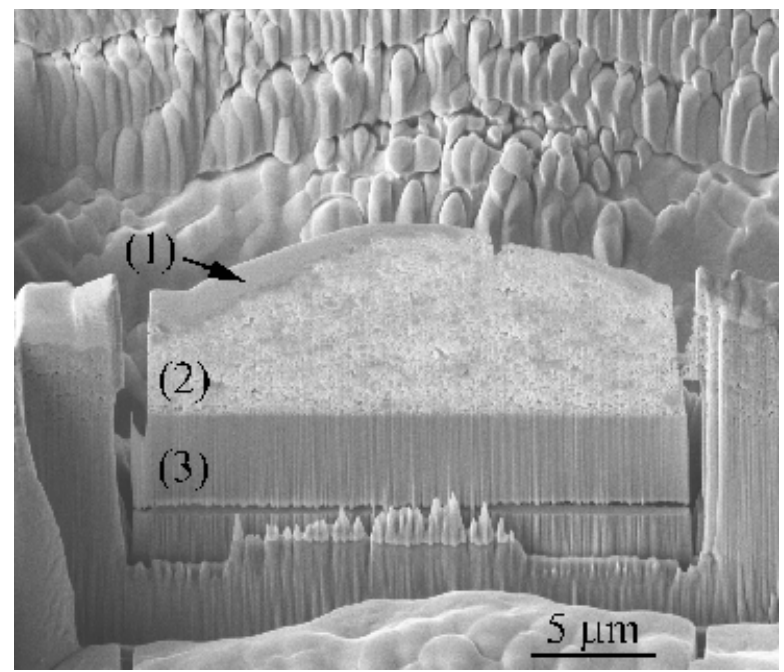

FIG. 2. SEM micrograph of the LO specimen. The layers labeled are: (1) Pt protective coating, (2) SiC nanoparticles, and (3) the $\mathrm{Si}$ substrate.

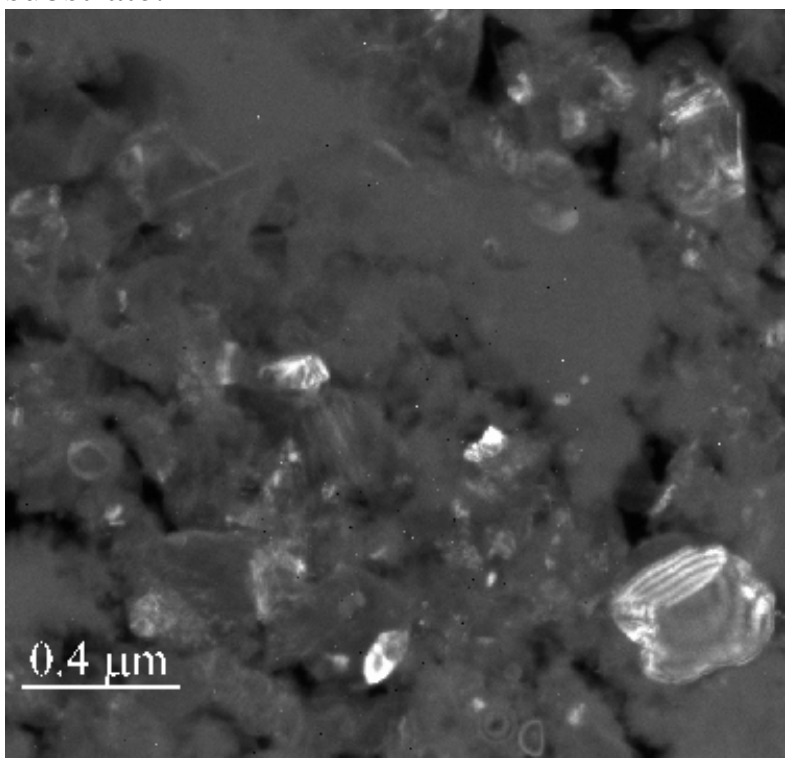

FIG. 4. Conical dark-field TEM micrograph showing the size of some of the nanoparticles. 